\title{
Comparison results for path-dependent options
}

\author{
Jan Bergenthum, Ludger Rüschendorf \\ Received: November 12, 2007; Accepted: April 4, 2008
}

\begin{abstract}
Summary: In this paper comparison results of convex type are established for several pathdependent options in some classes of semimartingale models. The options considered are some classes of lookback options, Asian and American options and barrier options. Comparison of the path-dependent options is based on ordering properties of the local characteristics of the underlying processes as well as on suitable propagation of convexity property. These properties allow a stochastic analysis of the basic linking process which establishes a link between the value processes in the underlying models. The linking process gives a unified tool to obtain comparison results for these path-dependent options. This paper extends and unifies several results in the literature.
\end{abstract}

\section{Introduction}

The problem of deriving ordering results for option prices has been addressed in several recent papers. For processes of diffusion type, for diffusions with jumps, for Lévy processes and PII processes, for exponential Lévy models and semimartingales several interesting comparison results in particular for European options have been obtained by various methods (see El Karoui et al. (1998), Hobson (1998), Bellamy and Jeanblanc (2000), Henderson (2000), Gushchin and Mordecki (2002), Bergenthum and Rüschendorf (2006, 2007a)). A main motivation for these results comes from the comparison of European options with respect to different pricing measures and from the problem of determining nontrivial price bounds. But similar comparison results are also of interest in various other areas as in complex networks or in insurance models.

In this paper we consider ordering results with respect to the (increasing) convex order for several classes of path-dependent options. The applications include examples of lookback options, Asian options, American options, and barrier options. Our results are of the type that certain ordering and convexity conditions on the underlying processes imply ordering results for path-dependent options of convex type. For (exponential) semimartingales it has been established in the papers mentioned above that comparison of the local characteristics and a propagation of convexity property imply convex ordering results for European options. In the case of (exponential) Lévy processes one obtains even ordering of the finite dimensional distributions. We remark that in the case of exponential

AMS 2000 subject classification: Primary: 62P05, 60E15; Secondary: 65C30

Key words and phrases: Path dependent options, lookback option, convex order, Lévy process, semimartingale 
Lévy processes $Y_{t}=\exp \left(X_{t}\right)$ increasing convex ordering results for the Lévy process $X$ imply increasing convex ordering results for the exponential Lévy process $Y$. It is typically more simple to give ordering conditions for $X$. By the previous remark however these imply ordering results for the exponential Lévy models which are the more relevant models for the financial applications (see [6]).

In Section 2 we combine finite dimensional ordering results as mentioned above with some closedness properties of orderings under weak convergence to obtain ordering results for path-dependent options like lookback options or options of Asian type for Lévy resp. exponential Lévy models. In Section 3 we extend these results for options of Asian type to the more general class of PII-models. Here instead of finite dimensional ordering of the underlyings we use Kolmogorov's backward equation for the gain function of the Asian option to derive the comparison results. We then generalize this approach to some classes of semimartingales by introducing a two dimensional Markov structure for the Asian options. In Section 4 we extend the approach introduced in El Karoui et al. (1998) and Bellamy and Jeanblanc (2000) for the comparison of American options in diffusion type models to more general classes of semimartingales. This approach is based on a characterization of American options by variational inequalities. Finally, in Section 5 we consider barrier options and give extensions of some results of Eriksson $(2004,2006)$ to more general models.

In our paper we consider the convex or increasing convex orders defined for r.v.s $X$, $Y$ by

$$
X \leq_{\text {icx }} Y \text { resp. } X \leq_{\mathrm{cx}} Y
$$

if $E f(X) \leq E f(Y)$ for all increasing convex resp. convex functions $f$ such that the integrals exist. Definition (1.1) can be stated in the same form for random vectors $X$, $Y$. For processes $S^{(1)}, S^{(2)}, S^{(i)}=\left(S_{t}^{(i)}\right)_{0 \leq t \leq T}$ we consider the corresponding product ordering of the finite dimensional distributions and define

$$
\left(S^{(1)}\right) \leq_{\text {icx }}\left(S^{(2)}\right) \quad \operatorname{resp} . \quad\left(S^{(1)}\right) \leq_{\mathrm{cx}}\left(S^{(2)}\right)
$$

if for all $0 \leq t_{1}<t_{2}<\cdots<t_{m} \leq T$ holds

$$
E f\left(S_{t_{1}}^{(1)}, \ldots, S_{t_{m}}^{(1)}\right) \leq E f\left(S_{t_{1}}^{(2)}, \ldots, S_{t_{m}}^{(2)}\right),
$$

for all functions $f$ which are increasing convex (resp. convex) in the $m$ components and such that the integrals exist. Similar product orderings $\leq_{\mathcal{F}}$ can be introduced for general function classes $\mathcal{F}$, replacing the class of convex functions (see [23, 25]). The product ordering in (1.3) is also called componentwise (increasing) convex ordering and denoted by $S^{(1)} \leq_{\text {ccx }} S^{(2)}$ resp. $S^{(1)} \leq_{\text {iccx }} S^{(2)}$. Since we would like to consider this product ordering also for other classes $\mathcal{F}$ we stick to the notation as above. The product odering in (1.2) is stronger than the multivariate (increasing) convex ordering defined via the class $\mathcal{F}_{\mathrm{cx}}=\mathcal{F}_{\mathrm{cx}}^{m}$ of all convex functions $f: \mathbb{R}^{m} \rightarrow \mathbb{R}$.

For time homogeneous Markov processes $S^{(1)}, S^{(2)}$ with transition kernels $Q^{(1)}, Q^{(2)}$ there is a simple sufficient condition for finite dimensional ordering:

$$
\left(S^{(1)}\right) \leq \mathcal{F}\left(S^{(2)}\right)
$$


holds if $S_{0}^{(1)} \leq \mathcal{F} S_{0}^{(2)}$ and if a $\leq \mathcal{F}$-monotone transition kernel $\left(Q_{t}\right)$ exists which separates $Q^{(1)}$ and $Q^{(2)}$, i.e. for all $x$ and $t>0$ holds

$$
Q_{t}^{(1)}(x, \cdot) \leq \mathcal{F} Q_{t}(x, \cdot) \leq \mathcal{F} Q_{t}^{(2)}(x, \cdot),
$$

(see [5, Proposition 3.1]). $Q$ is $\leq \mathcal{F}$-monotone if $f \in \mathcal{F}$ implies that $Q_{t} f(x)=\int f(y)$ $Q_{t}(x, d y) \in \mathcal{F}$ for all $t \geq 0$. This separation lemma applies in particular to Lévy processes and yields for them finite dimensional ordering results (see [5, 6]). For a general introduction to stochastic orderings and its applications we refer to Müller and Stoyan (2002).

\section{Lookback options}

In this section we consider lookback options that have path-dependent payoffs of the form

$$
g\left(\sup _{t \leq T} S_{t}\right)
$$

for an increasing convex function $g$. Throughout this section we consider càdlàg processes $S$ on $[0, T]$.

There are few results on orderings of lookback options in the literature. By a classical result of Blackwell and Dubins on stochastic ordering the supremum $L_{T}=\sup _{t \leq T} S_{t}$ of any martingale with final distribution $\mu$ of $S_{T}$ is bounded above in stochastic order by the Hardy-Littlewood transform of the law of $S_{T}$. This leads to a model independent upper bound for the lookback options in terms of a transform of the European option $g\left(S_{T}\right)$. A universal lower bound is given by the European option. Hobson (1998) gives trading strategies under which these bounds are attained. Henderson (2000) establishes stochastic comparison of the supremum of a stochastic volatility model and the supremum of a time-homogeneous diffusion model, making use of a comparison result for diffusions in Hajek (1985). Večeř and Xu (2004) give a counterexample to show that the result of Hajek (1985) does in general not apply to Poisson models.

The ordering of lookback options (and other path-dependent options) for processes $S^{(1)}, S^{(2)}$ is particularly simple, when ordering of the finite dimensional distributions is available like in the case of homogeneous Markov processes (see (1.5)). A general principle in stochastic ordering allows to infer from finite dimensional ordering results for processes $S^{(1)}, S^{(2)}$ and some integrability conditions the ordering of continuous functionals of the processes. This leads in the case of the lookback options to the following result.

Proposition 2.1 Let $\left(S_{t}^{(i)}\right)_{t \in[0, T]}, i=1,2$, be one-dimensional processes with $S_{0}^{(i)} \geq 0$ and $E \sup _{t \leq T}\left(S_{t}^{(i)}\right)<\infty$.

$$
\text { If }\left(S^{(1)}\right) \leq_{\mathrm{icx}}\left(S^{(2)}\right), \quad \text { then } \sup _{t \leq T} S_{t}^{(1)} \leq_{\mathrm{icx}} \sup _{t \leq T} S_{t}^{(2)} .
$$


Proof: For $m \in \mathbb{N}$ and $0 \leq t_{1}<\cdots<t_{m} \leq T$ the ordering $\left(S^{(1)}\right) \leq_{\text {icx }}\left(S^{(2)}\right)$ implies that $\left(S_{t_{1}}^{(1)}, \ldots, S_{t_{m}}^{(1)}\right) \leq_{\text {icx }}\left(S_{t_{1}}^{(2)}, \ldots, S_{t_{m}}^{(2)}\right)$. We introduce approximations $S_{n}^{(i)}$ of $S^{(i)}$ defined as step functions for partitions $0=t_{n, 0}<t_{n, 1}<\cdots<t_{n, k_{n}}=T$

$$
S_{n, t}^{(i)}= \begin{cases}S_{t_{n, j}}^{(i)}, & \text { if } t_{n, j} \leq t<t_{n, j+1}, \\ S_{n, T}^{(i)}, & \text { if } t=T .\end{cases}
$$

Assuming that $\max \left(t_{n, j+1}-t_{n, j}\right) \rightarrow 0$ we obtain convergence in $D[0, T]$

$$
S_{n}^{(i)} \stackrel{\mathcal{L}}{\longrightarrow} S^{(i)}
$$

As consequence also $\sup _{0 \leq t \leq T} S_{n, t}^{(i)} \stackrel{\mathcal{L}}{\longrightarrow} \sup _{0 \leq t \leq T} S_{t}^{(i)}$, since sup is a continuous functional on $D[0, T]$. In order to obtain the comparison result in (2.2) w.r.t. increasing convex ordering $\leq_{\text {icx }}$ it is enough to establish

$$
E \sup _{0 \leq t \leq T} S_{n, t}^{(i)} \longrightarrow E \sup _{0 \leq t \leq T} S_{t}^{(i)}
$$

(see [13, Satz 3.1]). As $\sup _{t \leq T} S_{n, t}^{(i)} \leq \sup _{t \leq T} S_{t}^{(i)}$ for all $n \in \mathbb{N}$, we obtain by assumption that the sequences $\left(\sup _{t \leq T} S_{n, t}^{(i)}\right)$ are uniformly integrable and thus (2.4) follows.

Various criteria that yield finite dimensional ordering of Lévy processes $X^{(1)}, X^{(2)}$ w.r.t. the increasing convex order have been established in [6]. Basically ordering of the Lévy measures and of the initial distributions implies ordering of the finite dimensional distributions of the Lévy processes w.r.t. the product ordering. By Proposition 2.1 this implies ordering of the lookback option of the Lévy processes. This ordering results for Lévy processes $X^{(i)}$ however also implies the corresponding ordering for exponential Lévy models $S^{(i)}=\exp \left(X^{(i)}\right)$.

Corollary 2.2 (Ordering of lookback options in exponential models) Let $S^{(i)}=$ $\exp \left(X^{(i)}\right), i=1,2$ be exponential models with $X_{0}^{(i)}=0$ and $E \sup _{t \leq T} \exp \left(X_{t}^{(i)}\right)<\infty$. If finite dimensional ordering $\left(X^{(1)}\right) \leq_{\mathrm{icx}}\left(X^{(2)}\right)$ holds for the basic processes $X^{(i)}$, then it also holds for the lookback options of the exponential models $S^{(i)}$,

$$
\sup _{t \leq T} S_{t}^{(i)} \leq \mathrm{icx} \sup _{t \leq T} S_{t}^{(2)} .
$$

Proof: Since exp is a increasing convex function we have that also the exponential processes are ordered $\left(S^{(1)}\right) \leq_{\text {icx }}\left(S^{(2)}\right)$. Using that $\exp \left(\sup _{t \leq T} X_{t}^{(i)}\right)=\sup _{t \leq T} \exp \left(X_{t}^{(i)}\right)$, we obtain (2.5) as consequence of Proposition 2.1.

Remark 2.3 The ordering results in Proposition 2.1, Corollary 2.2 are not specific for the lookback option. Consider processes $S^{(i)}=\left(S_{t}^{(i)}\right)$ and the corresponding approximations 
$S_{n}^{(i)}=\left(S_{n, t}^{(i)}\right)$ as in Proposition 2.1 and in its proof. Let $H: \mathrm{D}[0, T] \rightarrow \mathbb{R}$ be a continuous increasing, convex functional such that

$$
H\left(S_{n}^{(i)}\right)=H_{n}\left(\left(S_{n, t_{n, j}}^{(i)}\right)\right)
$$

for some increasing convex function $H_{n}$ on $\mathbb{R}^{k_{n}}, H_{n} \in \mathcal{F}_{\mathrm{icx}}, i=1,2$ and where $\left(t_{n, j}\right)$ is a partition of $[0, T]$ as in the definition of $S_{n}^{(i)}$. Assume that, for some $Y \in L^{1}$, $Y \leq H\left(S_{n}^{(i)}\right)$ and $H\left(S^{(i)}\right) \in L^{1}, \quad i=1,2, n \in \mathbb{N}$. Then

$$
\left(S^{(1)}\right) \leq_{\text {icx }}\left(S^{(2)}\right) \text { implies } H\left(S^{(1)}\right) \leq_{\text {icx }} H\left(S^{(2)}\right) .
$$

A representation as in (2.6) holds for several options as for lookback options $H(S)=$ $g\left(\sup _{0 \leq t \leq T} S_{t}\right)$, for Asian options $H(S)=g\left(\frac{1}{T} \int_{0}^{T} S_{t} d t\right), g \in \mathcal{F}_{\text {icx }}$, for barrier options $H(S)=g\left(S_{T}\right) \mathbb{1}_{\left\{\sup _{0 \leq t \leq T} S_{t} \geq \beta\right\}}$ and several others. By Corollary 2.2 the ordering assumption on the exponential model $S^{(i)}=\exp \left(X^{(i)}\right)$ is implied by corresponding finite dimensional ordering of $X^{(i)}$. As consequence we obtain in particular for (exponential) Lévy models easy to verify sufficient conditions for the (increasing) convex ordering of lookback options, of Asian options and of barrier options with constant barrier. This applies in particular to several relevant exponential Lévy models in mathematical finance (see $[4,6])$.

\section{Asian options}

In this section we consider Asian options for some classes of univariate or multivariate underlyings $S^{(i)}$. Our aim is to obtain ordering results for the comparison of Asian options of processes $S^{(1)}, S^{(2)}$ as in (2.7) but without posing the assumption of finite dimensional ordering for $S^{(1)}, S^{(2)}$, which may be difficult to verify except in homogeneous Markov processes or their exponential versions. We also consider the comparison of two Asian options with the same underlying $S$ but with respect to different averaging time intervals, i.e. to compare

$$
\frac{1}{\vartheta_{1}} \int_{T-\vartheta_{1}}^{T} S_{t} d t \text { and } \frac{1}{\vartheta_{2}} \int_{T-\vartheta_{2}}^{T} S_{t} d t \quad \text { for } \vartheta_{1}<\vartheta_{2} \text {. }
$$

Thus we consider the dependence on $\vartheta$ for terminal payoffs of the form

$$
g\left(\frac{1}{\vartheta} \int_{T-\vartheta}^{T} S_{t} d t\right), \quad g \in \mathcal{F}_{\mathrm{cx}}
$$

where $S$ is the (discounted) value of an underlying, w.r.t. a martingale measure.

There are some ordering results for prices of Asian options with continuous averaging in the literature. El Karoui et al. (1998) establish by stochastic analysis that Asian option prices in a univariate diffusion model are bounded above by the corresponding European option prices. This comparison result can be obtained alternatively in a simple way for any 
model $S$ in which $S_{t} \leq_{\mathrm{cx}} S_{T}$ for all $t \leq T$. Under this comparison assumption Jensen's inequality implies for $g \in \mathcal{F}_{\mathrm{cx}}$

$$
E g\left(\frac{1}{\vartheta} \int_{T-\vartheta}^{T} S_{u} d u\right) \leq \frac{1}{\vartheta} \int_{T-\vartheta}^{T} E g\left(S_{u}\right) d u \leq E g\left(S_{T}\right) .
$$

Bellamy and Jeanblanc (2000) establish that the lower bound of an Asian option price in a univariate diffusion with jumps model is given by the Asian option price under the corresponding Black-Scholes model with the same diffusion coefficient.

We derive two different kinds of ordering results for Asian options with respect to continuous averaging. At first we establish that for PII models Asian option prices are decreasing in the length of the averaging interval $[T-\vartheta, T]$. The highest price is obtained for $\vartheta \rightarrow 0$ (cp. (3.3)). For the proof of this result we make use of the value process of the average process with averaging interval $[T-\vartheta, T]$ given by

$$
A_{t}^{\vartheta}:=E\left(\frac{1}{\vartheta} \int_{T-\vartheta}^{T} S_{u} d u \mid \mathcal{A}_{t}\right), \quad 0 \leq t \leq T,
$$

where $\left(\Omega, \mathcal{A},\left(\mathcal{A}_{t}\right)\right)$ denotes the underlying stochastic basis. Then, we establish for PII models and for some semimartingale models that Asian option prices are ordered, if the local characteristics of the underlyings $S^{(i)}$ are ordered in an appropriate sense. For a semimartingale $S$ we denote by $S \sim(b, c, K)_{\text {id }}$, that $S$ has (differential) drift, diffusion and jump characteristics $b, c, K$ w.r.t. the truncation function $h=$ id. We assume throughout this paper that differential characteristics exist and that the identity can be chosen as truncation function.

In general the drift, diffusion, and jump characteristics are time-dependent and we use the notation $b=b_{u}=b(u), c=c_{u}=c(u), K=K_{u}=K(u)$. For Markovian semimartingales we also use the functional form $b=b(u, s), c=c(u, s), K=K_{u}(s, d x)$. For general reference to (differential) characteristics we refer to Jacod and Shiryaev (2003).

At first, we compute the (differential) characteristics of the value process $A^{\vartheta}$.

Lemma 3.1 (Characteristics of the value process $\left.\boldsymbol{A}_{t}^{\vartheta}\right) \quad$ Let $S_{t} \sim\left(0, c_{t}^{S}, K_{t}^{S}\right)$ be a martingale. Then the value process $A^{\vartheta} \sim\left(b_{t}^{A^{\vartheta}}, c_{t}^{A^{\vartheta}}, K_{t}^{A^{\vartheta}}\right)_{\mathrm{id}}$ in (3.4) has characteristics

$$
\begin{aligned}
b_{t}^{A^{\vartheta}} & =0, \\
c_{t}^{A^{\vartheta}} & =c_{t}^{S}\left(\mathbb{1}_{[0, T-\vartheta)}(t)+\left(\frac{T-t}{\vartheta}\right)^{2} \mathbb{1}_{[T-\vartheta, T]}(t)\right), \\
K_{t}^{A^{\vartheta}}(G) & =\int_{\mathbb{R}^{d}} \mathbb{1}_{G}(\alpha(t) y) K_{t}^{S}(d y), \quad G \in \mathcal{B}^{d},
\end{aligned}
$$

where $\alpha(t)=\mathbb{1}_{[0, T-\vartheta)}(t)+\frac{T-t}{\vartheta} \mathbb{1}_{[T-\vartheta, T]}(t)$. 
Proof: The value process $A^{\vartheta}$ is a martingale by definition, thus $b_{t}^{A^{\vartheta}}=0$. From Fubini's theorem and the martingale property of $S$ it follows that $A_{t}^{\vartheta}$ has a representation

$$
\begin{aligned}
A_{t}^{\vartheta} & =\frac{1}{\vartheta} \int_{T-\vartheta}^{T} E\left(S_{u} \mid \mathcal{A}_{t}\right) d u \\
& =S_{t} \mathbb{1}_{[0, T-\vartheta)}(t)+\frac{1}{\vartheta}\left(\int_{T-\vartheta}^{t} S_{u} d u+(T-t) S_{t}\right) \mathbb{1}_{[T-\vartheta, T]}(t) .
\end{aligned}
$$

As $\int_{T-\vartheta}^{t} S_{u} d u$ is continuous and of finite variation it follows that the quadratic characteristic of $A_{t}^{\vartheta}$ is given by $\left\langle A^{\vartheta}\right\rangle_{t}=\langle S\rangle_{t} \mathbb{1}_{[0, T-\vartheta)}(t)+\left(\frac{T-t}{\vartheta}\right)^{2}\langle S\rangle_{t} \mathbb{1}_{[T-\vartheta, T]}(t)$, hence the differential quadratic characteristic $c_{t}^{A^{\vartheta}}$ is of the stated form. As the jumps of $A^{\vartheta}$ are of the form $\Delta A_{t}^{\vartheta}=\alpha(t) \Delta S_{t}=\Delta(\alpha \cdot S)_{t}$, it follows that the jump compensator $v^{A^{\vartheta}}$ of $A_{t}^{\vartheta}$ is given by $v^{A^{\vartheta}}(\omega ;[0, t] \times G)=\int_{[0, t] \times \mathbb{R}^{d}} \mathbb{1}_{G}(\alpha(u) y) v^{S}(\omega ; d u, d y)$, hence the differential jump characteristic $K_{t}^{A^{\vartheta}}$ is as stated in (3.5).

From Lemma 3.1 it is seen that for a Markovian underlying process $S$ with dependent increments, the value process $A^{\vartheta}$ is not Markovian, as in this case $c^{A^{\vartheta}}$ and $K^{A^{\vartheta}}$ depend on $S_{t-}$. For the approach used in this paper it is however essential that one of the processes to be compared is Markovian. There are two possibilities to circumvent this problem. If $S$ is assumed to be a process with independent increments (PII), i.e. its characteristics are deterministic functions of time, then also $A^{\vartheta}$ is a PII. An alternative way is to enlarge the space of underlyings by $A^{\vartheta}$, as the two dimensional process $\left(S, A^{\vartheta}\right)$ is Markovian.

For the following comparison result we assume that $S \sim\left(0, c^{S}(t), K^{S}(t, \cdot)\right)$ is a $d$-dimensional PII martingale, hence the value process $A^{\vartheta} \sim\left(0, c^{A^{\vartheta}}(t), K^{A^{\vartheta}}(t, \cdot)\right)$ of the Asian option with averaging interval $[T-\vartheta, T]$ and convex payoff function $g$ also is a PII martingale. The corresponding backward function for the Asian option

$$
\mathcal{G}^{A^{\vartheta}}(t, a)=E\left(g\left(A_{T}^{\vartheta}\right) \mid A_{t}^{\vartheta}=a\right)
$$

satisfies under some regularity conditions the Kolmogorov-backward equation (see [5])

$$
\mathrm{D}_{t} \mathcal{G}^{A^{\vartheta}}(t, a)+\frac{1}{2} \sum_{i, j \leq d} \mathrm{D}_{i j}^{2} \mathcal{G}^{A^{\vartheta}}(t, a) c^{A^{\vartheta}}(t)+\int\left(\Lambda \mathcal{G}^{A^{\vartheta}}\right)(t, a, y) K^{A^{\vartheta}}(t, d y)=0,
$$

where $\left(\Lambda \mathcal{G}^{A^{\vartheta}}\right)(t, a, y)=\mathcal{G}^{A^{\vartheta}}(t, a+y)-\mathcal{G}^{A^{\vartheta}}(t, a)-\sum_{i \leq d} \mathrm{D}_{i} \mathcal{G}^{A^{\vartheta}}(t, a) y^{i}$

In the following theorem we establish that Asian option prices for PII processes are decreasing in the length of the averaging intervals. Thus the prices are lowest for the largest averaging interval $[0, T]$. The highest price is attained for $\vartheta \rightarrow 0$, i.e., for European options (cp. (3.3)).

Theorem 3.2 (Ordering of Asian option prices in the length of the averaging interval) Let $g: \mathbb{R}^{d} \rightarrow \mathbb{R}, g \in \mathcal{F}_{\mathrm{cx}}$, and assume that $S \sim\left(0, c^{S}(t), K^{S}(t, \cdot)\right)_{\mathrm{id}}$ is a positive martingale with independent increments and Lévy kernels $K^{S}(t, \cdot)$ that satisfy $\int y K^{S}(t, d y)=0$, for all $t \in[0, T]$. Assume that $\mathcal{G}^{A^{\vartheta}} \in C^{1,2}\left([0, T] \times \mathbb{R}^{d}\right)$ and 
that $\mathcal{G}^{A^{\vartheta}}(t, a)$ satisfies the Kolmogorov backward equation (3.7). Let the linking process $\mathcal{G}^{A^{\vartheta}}\left(t, A^{\vartheta_{1}}\right)$ be lower bounded and integrable. Then for $0<\vartheta_{1}<\vartheta$ it holds true that

$$
E g\left(\frac{1}{\vartheta} \int_{T-\vartheta}^{T} S_{u} d u\right) \leq E g\left(\frac{1}{\vartheta_{1}} \int_{T-\vartheta_{1}}^{T} S_{u} d u\right) .
$$

Proof: The basic idea of the proof is similar to that in the proofs of the comparison theorems for European options in [5]. We establish that the linking process between $A^{\vartheta}$ and $A^{\vartheta_{1}}$ defined as $\mathcal{G}^{A^{\vartheta}}\left(t, A_{t}^{\vartheta_{1}}\right)$ is a supermartingale. From Itô's formula and the Kolmogorov backward equation it follows that $\mathcal{G}^{A^{\vartheta}}\left(t, A_{t}^{\vartheta_{1}}\right)=\mathcal{G}^{A^{\vartheta}}\left(t, A_{0}^{\vartheta_{1}}\right)+M_{t}+V_{t}^{A^{\vartheta}}$, where $M_{t}$ is a local martingale and

$$
\begin{aligned}
V_{t}^{A^{\vartheta}}=\int_{0}^{t}\{ & \frac{1}{2} \sum_{i, j \leq d} \mathrm{D}_{i j}^{2} \mathcal{G}^{A^{\vartheta}}\left(u, A_{u}^{\vartheta_{1}}\right)\left(c^{A^{\vartheta_{1}}}(u)-c^{A^{\vartheta}}(u)\right) \\
& \left.+\int_{\mathbb{R}^{d}}\left(\Lambda \mathcal{G}^{A^{\vartheta}}\right)\left(u, A_{u-}^{\vartheta_{1}}, y\right)\left(K^{A^{\vartheta_{1}}}(u, d y)-K^{A^{\vartheta}}(u, d y)\right)\right\} d u .
\end{aligned}
$$

As $A^{\vartheta_{1}}$ and $A^{\vartheta}$ are PII, convexity of $g$ is propagated to $\mathcal{G}^{A^{\vartheta}}(t, \cdot)$ (see [4]). Hence it remains to establish suitable ordering of the characteristics of $A^{\vartheta_{1}}$ and $A^{\vartheta}$. From Lemma 3.1 it follows that

$$
\begin{aligned}
c^{A^{\vartheta}}(t) & =c^{S}(t) \mathbb{1}_{[0, T-\vartheta]}(t)+\left(\frac{T-t}{\vartheta}\right)^{2} c^{S}(t) \mathbb{1}_{[T-\vartheta, T]}(t) \\
& \operatorname{spsd} c^{S}(t) \mathbb{1}_{\left[0, T-\vartheta_{1}\right]}(t)+\left(\frac{T-t}{\vartheta_{1}}\right)^{2} c^{S}(t) \mathbb{1}_{\left[T-\vartheta_{1}, T\right]}(t) \\
& =c^{A^{\vartheta_{1}}}(t),
\end{aligned}
$$

here $\leq$ psd denotes positive semidefinite ordering. Lemma 3.1 also implies

$$
\begin{gathered}
\int f(t, y) K^{A^{\vartheta}}(t, d y)=\left\{\begin{array}{ll}
\int f(t, y) K^{S}(t, d y), & t<T-\vartheta \\
\int f\left(t, \frac{T-t}{\vartheta} y\right) K^{S}(t, d y), & t \geq T-\vartheta
\end{array}\right. \text { and } \\
\int f(t, y) K^{A^{\vartheta}}(t, d y)= \begin{cases}\int f(t, y) K^{S}(t, d y), & t<T-\vartheta_{1}, \\
\int f\left(t, \frac{T-t}{\vartheta} y\right) K^{S}(t, d y), & t \geq T-\vartheta_{1} .\end{cases}
\end{gathered}
$$

As for a random vector $X$ with $E X=0$ and for $\alpha \in[0,1]$ it holds true that $\alpha X \leq_{\mathrm{cx}} X$, it follows from the assumption $\int y K^{S}(t, d y)=0$ that

$$
\int f(t, y) K^{A^{\vartheta}}(t, d y) \leq \int f(t, y) K^{A^{\vartheta} 1}(t, d y), \quad \forall t \in[0, T],
$$

for all $f:[0, T] \times \mathbb{R}^{d} \rightarrow \mathbb{R}$ such that $f(t, \cdot) \in \mathcal{F}_{\mathrm{cx}}$. As consequence we obtain that the linking process $\mathcal{G}^{A^{\vartheta}}\left(t, A_{t}^{\vartheta_{1}}\right)$ is a supermartingale. 
This implies the comparison result using the crucial role of the linking process.

$$
E g\left(A_{T}^{\vartheta_{1}}\right)=E G^{A^{\vartheta}}\left(T, A_{T}^{\vartheta_{1}}\right) \leq E G^{A^{\vartheta}}\left(0, A_{0}^{\vartheta_{1}}\right)=E g\left(A_{T}^{\vartheta}\right) .
$$

Based on the linking process and Lemma 3.1 we also obtain ordering properties of Asian option prices on two multivariate PII underlyings $S^{(i)}, i=1,2$, in dependence on the local characteristics of $S^{(i)}$. The value processes of the Asian options are denoted by

$$
A_{t}^{(i)}:=\frac{1}{T} E\left(\int_{0}^{T} S_{u}^{(i)} d u \mid \mathcal{A}_{t}\right) .
$$

The Kolmogorov backward equation for the value function $\mathcal{G}^{(2)}(t, a)=E\left(g\left(A_{T}^{(2)}\right) \mid A_{t}^{(2)}\right.$ $=a)$ is of the form

$$
\mathrm{D}_{t} \mathcal{G}^{(2)}(t, a)+\frac{1}{2} \sum_{i, j \leq d} \mathrm{D}_{i j}^{2} \mathcal{G}^{(2)}(t, a) c^{A^{(2)}}(t)+\int\left(\Lambda \mathcal{G}^{(2)}\right)(t, a, y) K^{A^{(2)}}(t, d y)=0 .
$$

Theorem 3.3 (Comparison of Asian option prices in the characteristics of the underlying) Let $S^{(i)} \sim\left(0, c^{S^{(i)}}(t), K^{S^{(i)}}(t, \cdot)\right)_{\mathrm{id}}, i=1,2$, be d-dimensional PII martingales and $g: \mathbb{R}^{d} \rightarrow \mathbb{R}, g \in \mathcal{F}_{\mathrm{cx}}$. Assume that $\mathcal{G}^{(2)} \in C^{1,2}\left([0, T] \times \mathbb{R}^{d}\right)$ and the value function $\mathcal{G}^{(2)}$ satisfies the Kolmogorov backward equation (3.12). If

$$
\begin{aligned}
c^{S^{(1)}}(t) & \leq \mathrm{psd} c^{S^{(2)}}(t) \quad \text { and } \\
\int f(t, y) K^{S^{(1)}}(t, d y) & \leq \int f(t, y) K^{S^{(2)}}(t, d y),
\end{aligned}
$$

for all t and all $f:[0, T] \times \mathbb{R}^{d} \rightarrow \mathbb{R}$, such that $f(t, \cdot) \in \mathcal{F}_{\mathrm{cx}}$, then

$$
E g\left(\frac{1}{T} \int_{0}^{T} S_{u}^{(1)} d u\right) \leq E g\left(\frac{1}{T} \int_{0}^{T} S_{u}^{(2)} d u\right) .
$$

Proof: The proof is similar to that of Theorem 3.2. We consider the linking process $\mathcal{G}^{(2)}\left(t, A_{t}^{(1)}\right)$ and derive from Itô's formula and the Kolmogorov backward equation an expansion similar to (3.9). (3.13) then follows from the supermartingale property of the linking process $\mathcal{G}^{(2)}\left(t, A_{t}^{(1)}\right)$ similarly as in (3.10) using the following ordering properties of the characteristics. By Lemma 3.1 it follows from $c^{S^{(1)}}(t) \leq \mathrm{psd} c^{S^{(2)}}(t)$ that

$$
c^{A^{(1)}}(t)=c^{S^{(1)}}(t)\left(\frac{T-t}{T}\right)^{2} \leq \text { psd } c^{S^{(2)}}(t)\left(\frac{T-t}{T}\right)^{2}=c^{A^{(2)}}(t) .
$$

Further, for the Lévy kernels $K^{A^{(i)}}$, Lemma 3.1 implies

$$
\int f(t, y) K^{A^{(i)}}(t, d y)=\int f\left(t, y \frac{T-t}{T}\right) K^{S^{(i)}}(t, d y) .
$$


For $g(t, y):=f\left(t, y \frac{T-t}{T}\right)$ it follows that $g(t, \cdot) \in \mathcal{F}_{\mathrm{cx}}$ and thus by the ordering assumptions on $K^{S^{(i)}}$ the ordering conditions for $K^{A^{(i)}}$ are fulfilled.

We next extend the ordering result in Theorem 3.3 in the one-dimensional case to more general martingales and compare the Asian options in the case of martingales $S$, $S^{*}$, where $S^{*}$ is is assumed to be Markovian. To indicate the Markovian property of the second process we use the notation $S, S^{*}$ instead of $S^{(1)}, S^{(2)}$ from here on. This needs a different approach which is directly based on the averaging processes $A, A^{*}$ (instead of the value processes $A^{(i)}$ ) defined by

$$
A_{t}:=\frac{1}{t} \int_{0}^{t} S_{u} d u \text { and } A_{t}^{*}=\frac{1}{t} \int_{0}^{t} S_{u}^{*} d u .
$$

$A, A^{*}$ are continuous processes, have paths of finite variation and satisfy

$$
d A_{t}=\frac{1}{t}\left(S_{t}-A_{t}\right) d t, \quad d A_{t}^{*}=\frac{1}{t}\left(S_{t}^{*}-A_{t}^{*}\right) d t .
$$

Hence $A, A^{*}$ are not Markovian, even if $S^{*}$ is Markovian. To obtain a Markovian upper bound candidate, we augment $A$ and $A^{*}$ by the underlyings $S$ and $S^{*}$, respectively. Then the augmented process $\left(S^{*}, A^{*}\right)$ is Markovian as $S^{*}$ is assumed to be Markovian. The value process for the Asian option with payoff $g\left(A_{T}^{*}\right)$ then takes the form

$$
\mathcal{G}^{A^{*}}(t, s, a)=E^{*}\left(g\left(A_{T}^{*}\right) \mid S_{t}^{*}=s, A_{t}^{*}=a\right)
$$

and satisfies the Kolmogorov-backward equation

$$
\begin{aligned}
0= & \mathrm{D}_{t} \mathcal{G}^{A^{*}}(t, s, a)+\mathrm{D}_{a} \mathcal{G}^{A^{*}}(t, s, a) \frac{1}{t}(s-a)+\frac{1}{2} \mathrm{D}_{s s}^{2} \mathcal{G}^{A^{*}}(t, s, a) c^{S^{*}}(t, s) \\
& +\int_{\mathbb{R}}\left(\mathcal{G}^{A^{*}}(t, s+y, a)-\mathcal{G}^{A^{*}}(t, s, a)-\mathrm{D}_{s} \mathcal{G}^{A^{*}}(t, s, a) y\right) K^{S^{*}}(t, s, d y),
\end{aligned}
$$

cp. Barraquand and Pudet (1996) for the continuous case. For the general case see [5, Lemma 2.1]. Now we can apply a similar expansion technique as in the proofs of Theorems 3.2, 3.3 to the extended linking process $\mathcal{G}^{A^{*}}\left(t, S_{t}, A_{t}\right)$ and obtain the following version of a comparison result of Asian option prices.

Theorem 3.4 (Comparison of Asian option prices in the characteristics of the underlying) Let $S \sim\left(0, c^{S}, K^{S}\right)_{\mathrm{id}}, S^{*} \sim\left(0, c^{S^{*}}(t, s), K^{S^{*}}(t, s, \cdot)\right)_{\mathrm{id}}$ be one-dimensional martingales, and additionally assume that $S^{*}$ is Markovian. Let $g: \mathbb{R} \rightarrow \mathbb{R}, g \in \mathcal{F}_{\mathrm{cx}}$ assume that the value process $\mathcal{G}^{A^{*}}(t, s, a) \in C^{1,2,1}([0, T] \times \mathbb{R} \times \mathbb{R})$ and that the linking process $\mathcal{G}^{A^{*}}\left(t, S_{t}, A_{t}\right)$ is lower bounded and integrable. Further assume the propagation of convexity condition $\mathcal{G}^{A^{*}}(t, \cdot, a) \in \mathcal{F}_{\mathrm{cx}}$ for all $0 \leq t \leq T$ and $a \in \mathbb{R}$ (see (3.16)). If

$$
\begin{aligned}
c_{t}^{S}(\omega) & \leq c^{S^{*}}\left(t, S_{t-}(\omega)\right) \quad \text { and } \\
\int f\left(t, S_{t-}(\omega), z\right) K_{\omega, t}^{S}(d z) & \leq \int f\left(t, S_{t-}(\omega), z\right) K_{t}^{S^{*}}\left(S_{t-}(\omega), d z\right),
\end{aligned}
$$


$\lambda \times Q$-a.e., for all $f:[0, T] \times \mathbb{R} \times \mathbb{R} \rightarrow \mathbb{R}_{+}$with $f(t, s, \cdot) \in \mathcal{F}_{\mathrm{cx}}$ such that the integrals exist, then

$$
E g\left(\frac{1}{T} \int_{0}^{T} S_{u} d u\right) \leq E g\left(\frac{1}{T} \int_{0}^{T} S_{u}^{*} d u\right)
$$

Proof: The main part of the proof is to establish that $\mathcal{G}^{A^{*}}\left(t, S_{t}, A_{t}\right)$ is a supermartingale. Due to Itô's formula and the form of the Kolmogorov-backward equation in (3.17) the crucial equation is

$$
\begin{aligned}
\mathcal{G}^{A^{*}}\left(t, S_{t}, A_{t}\right) & \\
= & \mathcal{G}^{A^{*}}\left(0, S_{0}, A_{0}\right)+M_{t}+\int_{0}^{t} \frac{1}{2} \mathrm{D}_{s s}^{2} \mathcal{G}^{A^{*}}\left(t, S_{u-}, A_{u-}\right)\left(c_{u}^{S}-c^{S^{*}}\left(u, S_{u-}\right)\right) d u \\
+\int_{0}^{t} \int_{\mathbb{R}} & \left(\mathcal{G}^{A^{*}}\left(u, S_{u-}+y, A_{u-}\right)-\mathcal{G}^{A^{*}}\left(u, S_{u-}, A_{u-}\right)\right. \\
& \left.\quad-\mathrm{D}_{s} \mathcal{G}^{A^{*}}\left(u, S_{u-}, A_{u-}\right) y\right)\left(K_{u}^{S}-K^{S^{*}}\left(u, S_{u-}, d y\right)\right) d u .
\end{aligned}
$$

Hence convexity of $\mathcal{G}^{A^{*}}(t, \cdot, a)$ and the orderings of the characteristics of $S$ and $S^{*}$ imply the result.

Remark 3.5 a) The assumption of lower boundedness and integrability of the linking value processes in the theorems above ensures that the local supermartingale property can be improved to the supermartingale property. An alternative sufficient condition for this conclusion is to assume uniform integrability of the linking process. This remark concerns also related results in the following part of this paper.

b) Reversing the ordering assumptions on the local characteristics we obtain in a similar way a lower bound for Asian option prices by those in a Markovian model. Here we need to establish the local submartingale property of the linking process and then have to use the corresponding integrability conditions.

\section{American options}

In this section we derive some ordering results for American option prices in semimartingale models. Parallel to the European case, the orderings are formulated in terms of the differential characteristics of the underlyings. We assume that interest rates exist, and also that stocks may pay dividend yields. In an equity model, in which interest rates are assumed to be higher than dividends and in a foreign exchange model, in which the domestic interest rate is assumed to be higher than the foreign interest rate, the prices of American and European call options coincide, whereas American put options yield higher prices than their European counterparts. Similarly, if the dividend yield in an equity model majorizes the interest rates or if in a foreign exchange model the domestic interest 
rate is lower than the foreign interest rate, then American and European put option prices are identical, whereas American call options yield higher prices than their European counterparts.

There are several comparison results for American options in the literature, most of which are derived as extensions of ordering results for European option prices. Pham (1997) derives properties of American option prices in a jump diffusion model, where the jump part is driven by a homogeneous Poisson random measure with finite intensity $\lambda$. Pham characterizes American option prices by a parabolic integro-differential free-boundary problem, and then obtains monotonicity of American put option prices in $\lambda$ and in the Girsanov jump parameter. El Karoui et al. (1998) establish upper and lower bounds for American put option prices in univariate stochastic volatility models. They use a variational inequality approach that is parallel to the stochastic calculus approach that is used to establish orderings for European type options. Hobson (1998) also considers continuous models. For a univariate diffusion he derives convexity of American put option prices in the underlying, and also obtains option price monotonicity in the diffusion coefficient. Parallel to the approach for European type options, he uses coupling arguments to derive the orderings. Bellamy and Jeanblanc (2000) establish that a lower bound of an American option price w.r.t. to a diffusion with jumps model is given by the price of an American option w.r.t. the corresponding generalized Black-Scholes model. As in the case of European options, the result is obtained by the stochastic calculus approach. Henderson and Hobson (2003) obtain ordering of American option prices in a diffusion with jumps model in the Girsanov parameter and then establish ordering of American option prices w.r.t. well established martingale measures by coupling techniques. In a univariate diffusion model, Ekström (2004) considers American options with payoff functions that satisfy certain growth conditions, which are especially satisfied by decreasing functions. To establish convexity of American option prices in the underlying, Ekström uses the notion of volatility time, a time change introduced in Janson and Tysk (2003), and also obtains monotonicity and continuity of the prices in the volatility.

In the following we establish a comparison result for American options where the underlyings are semimartingales one of them being Markovian. We state the comparison result for semimartingales in the basic case with interest rate $r>0$ and without dividend yield. In order to extend the variational approach in Bellamy and Jeanblanc (2000) to this more general situation it is useful to put the stopping problem for two processes in a frame, where we have one process $S$ but two probability measures $P$ and $P^{*}$. We assume that $(S, P)$ is a nonnegative semimartingale and $\left(S, P^{*}\right)$ is a nonnegative Markovian semimartingale. This framework is not very restrictive. If we want to compare two càdlàg semimartingales then we can equivalently consider the canonical process $S$ on $\mathrm{D}[0, T]$ with respect to the two distributions $P, P^{*}$ of the processes on $\mathrm{D}[0, T]$. We assume that $P, P^{*}$ are martingale measures for the discounted process $e^{-r t} S_{t}$. Denote by $X$ the stochastic logarithm of $S, X=\mathcal{L} \operatorname{og}(S)$. Then $X$ is a semimartingale with characteristics $X \sim\left(b_{u}, c_{u}, K_{u}\right)$ w.r.t. $P$ and $X \sim\left(b^{*}(u, s), c^{*}(u, s), K^{*}(u, s, d s)\right)$ w.r.t. $P^{*}$.

Let for a nonnegative convex function $g \in \mathcal{F}_{\mathrm{cx}}$

$$
\mathcal{G}_{\mathrm{Am}}(t):=\underset{\tau \in \mathcal{T}(t, T)}{\operatorname{ess} \sup } E e^{-r(\tau-t)}\left(g\left(S_{\tau}\right) \mid \mathcal{A}_{t}\right)
$$


denote the value process of the American option w.r.t. $P$ with filtration $\left(\mathcal{A}_{t}\right)$ generated by $S$ and with the set of stopping times $\mathcal{T}(t, T), t \leq \tau \leq T$. Similarly

$$
\mathcal{G}_{\mathrm{Am}}^{*}(t, s)=\underset{\tau \in \mathcal{T}(t, T)}{\operatorname{ess} \sup } E^{*} e^{-r(\tau-t)}\left(g\left(S_{\tau}\right) \mid S_{t}=s\right)
$$

denotes the value process w.r.t. the Markovian model. Under general conditions it has been established in the literature that $\mathcal{G}_{\mathrm{Am}}^{*}$ is characterized by the variational inequalities of Hamilton-Jacobi-Bellman type:

$$
\begin{aligned}
& \mathcal{G}_{\mathrm{Am}}^{*} \in C^{1,2}\left([0, T] \times \mathbb{R}^{1}\right), \\
& \max \left\{\mathrm{D}_{t} \mathcal{G}_{\mathrm{Am}}^{*}(t, s)+r s D_{s} \mathcal{G}_{\mathrm{Am}}(t, s)-r \mathcal{G}_{\mathrm{Am}}^{*}(t, s)+\frac{1}{2} c^{*}(t, s) s^{2} \mathrm{D}_{s s}^{2} \mathcal{G}_{\mathrm{Am}}^{*}(t, s)\right. \\
& \quad+\int\left\{\mathcal{G}_{\mathrm{Am}}^{*}(t, s(1+x))-\mathcal{G}_{\mathrm{Am}}^{*}(t, s)-s x \mathrm{D}_{s} \mathcal{G}_{\mathrm{Am}}^{*}(t, s)\right\} K^{*}(u, s, d x) \\
& \left.\quad g(s)-\mathcal{G}_{\mathrm{Am}}^{*}(t, s)\right\}=0 \\
& \text { and } \mathcal{G}_{\mathrm{Am}}^{*}(T, s)=g(s)
\end{aligned}
$$

While the characterization of $\mathcal{G}_{\mathrm{Am}}^{*}$ by the variational inequalities in (4.3) is quite general if derivatives are interpreted in a weak sense we need in the following the stronger assumption that $\mathcal{G}_{\mathrm{Am}}^{*} \in C^{1,2}$ in order to apply Itô's formula. This assumption is not satisfied in general for jump diffusions. The variational inequality and characterization of the value process has been studied for jump diffusions in Zhang (1994, Proposition 3.5).

We also need the propagation of convexity property:

$$
\text { For all } t \leq T \text { it holds that } \mathcal{G}_{\mathrm{Am}}^{*}(t, \cdot) \in \mathcal{F}_{\mathrm{cx}} \text {. }
$$

For jump diffusions this is established by Pham (1997). We denote by $\tau^{*}$ the optimal stopping time of the stopping problem w.r.t. $P^{*}$

$$
\tau^{*}=\inf \left\{0 \leq t \leq T ; g\left(S_{t}\right)=\mathcal{G}_{\mathrm{Am}}^{*}\left(t, S_{t}\right)\right\}
$$

For quasi-left-continuous processes $S$ w.r.t. $P^{*}$ it is known that an optimal stopping time exists and that $\tau^{*}$ as in (4.5) is optimal; in fact it coincides with the corresponding Snellstopping time (see Jamshidian (2006, Corollary 2.8)). Thus from now on we assume $S$ to be quasi-left-continuous. Then

$$
\mathcal{G}_{\mathrm{Am}}^{*}\left(\tau^{*}, S_{\tau^{*}}\right)=e^{-r \tau^{*}} g\left(S_{\tau^{*}}\right)\left[P^{*}\right] \text { and } E^{*} e^{-r \tau^{*}} g\left(S_{\tau^{*}}\right)=\mathcal{G}_{\mathrm{Am}}^{*}(0, s)
$$

For the following comparison result between semimartingales $S=\mathcal{E}(X)$ w.r.t. $P$ and w.r.t. $P^{*}$ we assume that $e^{-r t} S_{t}$ is a local martingale w.r.t. $P$ and a Markovian martingale w.r.t. $P^{*}$. From Yor's product formula

$$
\mathcal{E}(X) \mathcal{E}(Y)=\mathcal{E}(X+Y+[X, Y])
$$

follows that

$$
e^{-r t} S_{t}=\mathcal{E}(\tilde{X})
$$


with $\tilde{X} \sim\left(b_{u}-r, c_{u}, K_{u}\right)$ w.r.t. $P$ and $\tilde{X} \sim\left(b_{u}^{*}-r, c_{u}^{*}, K_{u}^{*}\right)$ w.r.t. $P^{*}$. Thus the local martingale property of $e^{-r t} S_{t}$ is equivalent to the conditions

$$
b_{u}-r+\frac{1}{2} c_{u}+\left(e^{x}-1-h(x)\right) * K_{u}=0
$$

and

$$
b_{u}^{*}-r+\frac{1}{2} c_{u}^{*}+\left(e^{x}-1-h(x)\right) * K_{u}^{*}=0,
$$

where $h$ is the truncation function and $*$ denotes the integral operation. We need the following convex ordering conditions on the differential characteristics of $X$ :

$$
\begin{aligned}
c_{t}(\omega) & \geq c^{*}\left(t, S_{t^{-}}(\omega)\right) \\
\int f\left(t, S_{t^{-}}(\omega), x\right) K_{\omega, t}(d x) & \geq \int f\left(t, S_{t^{-}}(\omega), x\right) K_{t}^{*}\left(S_{t^{-}}(\omega), d x\right)
\end{aligned}
$$

$\lambda \times P$ a.s. for all functions $f$ such that $f(t, s, \cdot) \in \mathcal{F}_{\mathrm{cx}}$ and this integral exist.

Theorem 4.1 (Comparison of American option prices) Let $S=\mathcal{E}(X)$ be a semimartingale w.r.t. $P$ with $X \sim\left(b_{u}, c_{u}, K_{u}\right)$ and a Markovian semimartingale w.r.t. $P^{*}$ with $X \sim\left(0, c^{*}(u, s), K_{u}^{*}(s, d x)\right)$. Assume that $e^{-r t} S_{t}$ is a local martingale w.r.t. $P$ and $P^{*}$ and $S_{0}=s \quad P+P^{*}$ almost surely. Let $g \in \mathcal{F}_{\mathrm{cx}}$ be a convex functional and assume that $\mathcal{G}_{\mathrm{Am}}^{*}\left(t, S_{t}\right)$ is bounded above and integrable. If the assumptions $(\mathrm{V}),(\mathrm{PC})$, and $(\mathrm{CO})$ hold true, then

$$
\mathcal{G}_{\mathrm{Am}}(0, s) \geq \mathcal{G}_{\mathrm{Am}}^{*}(0, s) .
$$

Proof: The basic role in the proof is played by the corresponding linking process $\mathcal{G}_{\mathrm{Am}}^{*}\left(t, S_{t}\right)$. Using that by assumption $(\mathrm{V}) \mathcal{G}_{\mathrm{Am}}^{*} \in C^{1,2}$, it follows from Itô's formula that w.r.t. $P$ the linking process $\mathcal{G}_{\mathrm{Am}}^{*}\left(t, S_{t}\right)$ connecting the American option prices in both models has the expansion

$$
\begin{aligned}
e^{-r t} \mathcal{G}_{\mathrm{Am}}^{*}\left(t, S_{t}\right)=\mathcal{G}_{\mathrm{Am}}^{*}(0, s)+ & M_{t} \\
+\int_{0}^{t} e^{-r u}\{ & \mathrm{D}_{u} \mathcal{G}_{\mathrm{Am}}^{*}\left(u, S_{u-}\right)+r S_{u-} \mathrm{D}_{s} \mathcal{G}_{\mathrm{Am}}^{*}\left(u, S_{u-}\right) \\
& \quad-r \mathcal{G}_{\mathrm{Am}}^{*}\left(u, S_{u-}\right)+\frac{1}{2} \mathrm{D}_{s s}^{2} \mathcal{G}_{\mathrm{Am}}^{*}\left(u, S_{u-}\right) c_{u} S_{u-}^{2} \\
& \left.+\int \Lambda G_{\mathrm{Am}}^{*}\left(u, S_{u_{-}} x\right) K_{u}(d x)\right\} d u \\
= & \mathcal{G}_{\mathrm{Am}}^{*}(0, s)+M_{t}+A_{t} .
\end{aligned}
$$

Here $\Lambda f(u, s, x)=f(u, s(1+x))-f(u, s)-\mathrm{D}_{s} f(u, s) x s, M_{t}$ is a local martingale w.r.t. $P, M_{t}=\int_{0}^{t} e^{-r u} \mathrm{D}_{s} \mathcal{G}_{\mathrm{Am}}^{*}\left(u, S_{u-}\right) d S_{u}$ and $A_{t}$ is a process of locally finite variation.

Now we obtain by definition of $\mathcal{G}_{\mathrm{Am}}$ and (4.6) that

$$
\mathcal{G}_{\mathrm{Am}}(0, s)=\sup _{\tau \in \mathcal{T}} E e^{-r \tau} g\left(S_{\tau}\right) \geq E e^{-r \tau^{*}} g\left(S_{\tau^{*}}\right)=E e^{-r \tau^{*}} \mathcal{G}_{\mathrm{A} m}^{*}\left(\tau^{*}, S_{\tau^{*}}\right) .
$$


Applying the variational condition $(\mathrm{V})$ this implies that on the interval $\left[0, \tau^{*}\right]$ the left-hand side of (4.3) is zero and thus on $\left[0, \tau^{*}\right]$

$$
\begin{aligned}
e^{-r t} & \mathcal{G}_{\mathrm{Am}}^{*}\left(t, S_{t}\right) \\
= & \mathcal{G}_{\mathrm{Am}}^{*}(0, s)+M_{t}+\int_{0}^{t} e^{-r u}\left\{\frac{1}{2} \mathrm{D}_{s s}^{2} \mathcal{G}_{\mathrm{Am}}^{*}\left(u, S_{u-}\right) S_{u-}^{2}\left(c_{u}-c^{*}\left(u, S_{u-}\right)\right)\right. \\
& \left.+\int \Lambda \mathcal{G}_{\mathrm{Am}}^{*}\left(u, S_{u-}, x\right)\left\{K_{u}(d x)-K_{u}^{*}\left(S_{u-}, d x\right)\right\}\right\} d u .
\end{aligned}
$$

By the propagation of convexity assumption (PC) and the ordering assumption (CO) $e^{-r t} \mathcal{G}_{\mathrm{Am}}^{*}\left(t, S_{t}\right)$ is a local submartingale on $\left[0, \tau^{*}\right]$ and thus by upper boundedness and integrability a submartingale w.r.t. $P$. As consequence we obtain

$$
\begin{aligned}
\mathcal{G}_{\mathrm{Am}}(0, s) & \geq E e^{-r \tau^{*}} \mathcal{G}_{\mathrm{Am}}^{*}\left(\tau^{*}, S_{\tau}^{*}\right) \\
& \geq \mathcal{G}_{\mathrm{Am}}^{*}(0, s) .
\end{aligned}
$$

Remark 4.2 a) As remarked above the propagation of convexity condition and the variational inequality have been studied in the case of jump diffusion processes.

b) In a similar way also lower bounds of American option prices by those in Markovian models can be derived.

c) An alternative way to prove of comparison results for American options in the undiscounted case is to consider in the first step comparison results for any stopping time $\tau$

$$
E g\left(S_{\tau}\right) \geq E^{*} g\left(S_{\tau}\right) .
$$

These are obtained as consequence of the comparison result for semimartingales in [5] since the stopped process $S^{\tau}$ is a semimartingale with characteristics given by the stopped versions of the characteristics of $S$. Several comparison results of this type are known also for nonconvex functions $g$. The new problems to consider are the smoothness condition and the propagation of ordering condition for the stopped processes. For the optimal stopping time $\tau=\tau^{*}$ of the $P^{*}$ stopping problem this is equivalent to the smoothness and propagation of ordering for $\mathcal{G}_{\mathrm{Am}}^{*}$ used above in the formulation of Theorem 4.1.

Statement (4.18) for $\tau=\tau^{*}$ implies

$$
\mathcal{G}_{\mathrm{Am}}(0, s)=\sup _{\tau} E g\left(S_{\tau}\right) \geq E g\left(S_{\tau^{*}}\right) \geq E^{*} g\left(S_{\tau^{*}}\right)=\mathcal{G}_{\mathrm{Am}}^{*}(0, s) .
$$

As a special case of application of Theorem 4.1 we consider two equity models $S^{(i)}$, $i=1,2$ with zero dividends and with positive constant interest rate $r>0$ (or more generally in the case that the interest rate $r$ is greater than the dividend rate $d$ ). We assume that the underlyings $S^{(i)}$ under the respective martingale measures are solutions of

$$
\frac{d S_{t}^{(i)}}{S_{t-}^{(i)}}=r d t+\sigma^{(i)} d W_{t}+\int_{(-1, \infty)} x\left(\mathrm{p}^{(i)}(d t, d x)-F^{(i)}(d x)\right) d t
$$


where $\sigma^{(i)}>0, W_{t}$ is a univariate Brownian motion, and $\mathrm{p}^{(i)}$ is a homogeneous Poisson random measure with finite compensator $F^{(i)}(d x) d t$. Hence the stochastic logarithm $X^{(i)}=\mathcal{L} \operatorname{og}\left(S^{(i)}\right)$ is a Lévy process with characteristics $X^{(i)} \sim\left(r, \sigma^{(i)^{2}}, F^{(i)}\right)_{\text {id }}$; equivalently $S^{(i)}$ is the stochastic exponential of $X^{(i)}, S^{(i)}=\mathcal{E}\left(X^{(i)}\right)$. The American option prices with payoff $g$ are given by

$$
\mathcal{G}_{\mathrm{Am}}^{(i)}(t, s)=\sup _{\tau \in \mathcal{T}_{t, T}} E\left(e^{-(\tau-t) r} g\left(S_{\tau}^{(i)}\right) \mid S_{t}^{(i)}=s\right) .
$$

The Hamilton-Jacobi-Bellman equation for $\mathcal{G}_{\mathrm{Am}}^{(2)}$ now takes the form

$$
\begin{aligned}
\mathcal{G}_{\mathrm{Am}}^{(2)} \in C^{1,2} \text { and } & \max \left\{\int\left(\mathcal{G}_{\mathrm{Am}}^{(2)}(t, s(1+x))-\mathcal{G}_{\mathrm{Am}}^{(2)}(t, s)-s x \mathrm{D}_{s} \mathcal{G}_{\mathrm{Am}}^{(2)}(t, s)\right) F^{(2)}(d x)\right. \\
& +\mathrm{D}_{t} \mathcal{G}_{\mathrm{Am}}^{(2)}(t, s)+r s \mathrm{D}_{s} \mathcal{G}_{\mathrm{Am}}^{(2)}(t, s)+\frac{\sigma^{(2)^{2}} s^{2}}{2} \mathrm{D}_{s s}^{2} \mathcal{G}_{\mathrm{Am}}^{(2)}(t, s) \\
& \left.-r \mathcal{G}_{\mathrm{Am}}^{(2)}(t, s), g(s)-\mathcal{G}_{\mathrm{Am}}^{(2)}(t, s)\right\}=0 .
\end{aligned}
$$

Zhang (1994, Proposition 3.5) establishes that (4.22) holds (in terms of the $\log$ arithm $\log S^{(2)}$ ) but in general the derivatives have to be taken in weak sense. The propagation of convexity property (PC) is established in Pham (1997). As consequence Theorem 4.1 implies:

Corollary 4.3 Let $g: \mathbb{R}_{+} \rightarrow \mathbb{R}_{+}$be convex and $S^{(i)}=\mathcal{E}\left(X^{(i)}\right), i=1,2$, with $X^{(i)} \sim$ $\left(r, \sigma^{(i)^{2}}, F^{(i)}\right)_{\mathrm{id}}, S_{0}^{(i)}=s$. Assume that the value function $\mathcal{G}_{\mathrm{Am}}^{(2)}$ satisfies Assumption $\left(\mathrm{V}^{\prime}\right)$ and that the linking process $\mathcal{G}_{\mathrm{Am}}^{(2)}\left(t, S_{t}^{(1)}\right)$ is bounded above and integrable. If the differential characteristics of $X^{(i)}$ satisfy the ordering conditions

$$
\begin{aligned}
\sigma^{(1)} & \geq \sigma^{(2)}, \\
\int f(x) F^{(1)}(d x) & \geq \int f(x) F^{(2)}(d x),
\end{aligned}
$$

for all non-negative $f \in \mathcal{F}_{\mathrm{cx}}$, then

$$
\mathcal{G}_{\mathrm{Am}}^{(1)}(0, s) \geq \mathcal{G}_{\mathrm{Am}}^{(2)}(0, s) .
$$

\section{Barrier options}

In this section we consider comparison of single-barrier options of European type without rebate in univariate models. The terminal payoff of a knock-out type barrier option on an underlying $S^{*}$ with barrier $\beta:[0, T] \rightarrow \mathbb{R}_{+}$and payoff function $g$ is given by

$$
g\left(S_{T}^{*}\right) \mathbb{1}_{\left\{\eta S_{t}^{*}>\eta \beta(t), \forall t \in[0, T]\right\}}, \quad \eta \in\{-1,1\} .
$$


For $\eta=1,(5.1)$ is the terminal payoff of a down-and-out barrier option, for $\eta=-1$ it is the terminal payoff of an up-and-out barrier option. We denote the value function of a down-and-out barrier option with barrier $\beta(t)$ by

$$
\mathcal{G}_{\text {out }}(t, s)=E\left(g\left(S_{T}^{*}\right) \mathbb{1}_{\left\{S_{u}^{*}>\beta(u), \forall u \in[t, T]\right\}} \mid S_{t}^{*}=s\right)=\mathcal{G}_{\text {out }}^{S^{*}}(t, s),
$$

and the value function of a up-and-out barrier option with barrier $\beta(t)$ by

$$
\mathcal{G}^{\text {out }}(t, s)=E\left(g\left(S_{T}^{*}\right) \mathbb{1}_{\left\{S_{u}^{*}<\beta(u), \forall u \in[t, T]\right\}} \mid S_{t}^{*}=s\right) .
$$

Similarly, a knock-in type barrier option has terminal payoff

$$
g\left(S_{T}^{*}\right) \mathbb{1}_{\left\{\eta S_{t}^{*}<\eta \beta(t), \exists t \in[0, T]\right\}}, \quad \eta \in\{-1,1\},
$$

where $\eta=1$ corresponds to a down-and-in barrier option, and $\eta=-1$ to an up-and-in barrier option, and corresponding value functions $\mathcal{G}_{\text {in }}(t, s)$ and $\mathcal{G}^{\text {in }}(t, s)$. A barrier option is said to be regular, if $g(\beta(T))=0$. For down-type barrier options we assume $S_{0}>\beta(0)$ and for up-type barrier options we assume $S_{0}<\beta(0)$.

It is of interest to distinguish the case with pure barrier $\beta(t)>0$ for all $t \in[0, T]$, and with discounted barrier $\beta(t) e^{-b(T-t)}, \beta>0$, where $b$ is the drift of the underlying $S$ under an equivalent martingale measure. In an equity model, if $S$ is a stock with continuously compounded annual dividend yield $d$, the drift is $b=r-d$, where $r$ is the risk-free rate of interest. In foreign exchange markets, where $S$ is an exchange rate between a domestic and a foreign currency, $b=r^{\text {dom }}-r^{\text {for }}$, where $r^{\text {dom }}$ and $r^{\text {for }}$ denote the domestic and the foreign interest rate, respectively.

For a one-dimensional diffusion model, Eriksson $(2004,2006)$ establishes monotonicity in the diffusion coefficient for the various types of barrier options that are given above. These ordering results depend on the drift coefficient of the underlying $S$. For $b=0$ it is shown that for regular down-and-out and up-and-out contracts and down-and-in and up-and-in contracts this monotonicity result holds true.

We assume that the value functions of the barrier options with underlying $S^{*}$ satisfy a PIDE that is of the form of the Kolmogorov-backward equation. Additionally to the terminal boundary condition, in the case of barrier option a boundary condition in the space variable occurs. In the sequel we discuss the case $b=0$, the cases $b \neq 0$ are treated similarly. Explicitly we consider comparison of down-and-out barrier options on onedimensional underlyings $S, S^{*}$ in a market model with zero interest rate. We assume that $S, S^{*}$ are positive martingales, $S \sim\left(0, c^{S}, K^{S}\right)_{\mathrm{id}}, S^{*} \sim\left(0, c^{S^{*}}\left(t, S_{t}^{*}\right), K^{S^{*}}\left(t, S_{t}^{*}, \cdot\right)\right)_{\mathrm{id}}$. The case $r>0$ can be dealt with similarly. We also assume that $S^{*}$ is Markovian. The value function $\mathcal{G}_{\text {out }}(t, s)$ satisfies the Kolmogorov backward equation

$$
\mathrm{D}_{t} \mathcal{G}_{\text {out }}(t, s)+\frac{1}{2} \mathrm{D}_{s s}^{2} \mathcal{G}_{\text {out }}(t, s) c^{S^{*}}(t, s)+\int\left(\Lambda \mathcal{G}_{\text {out }}\right)(t, s, y) K^{S^{*}}(t, s, d y)=0,
$$

on $(\beta, \infty)$, subject to boundary conditions

$$
\begin{aligned}
& \mathcal{G}_{\text {out }}(t, s)=0, \quad s \leq \beta \text { for all } t \in[0, T], \\
& \mathcal{G}_{\text {out }}(T, s)=g(s), \quad s>\beta,
\end{aligned}
$$

cp. Cont et al. (2004). We obtain the following ordering result for down-and-out barrier options. 
Theorem 5.1 (Convex ordering of barrier options, upper bound) Let $S \sim\left(0, c^{S}\right.$, $\left.K^{S}\right)_{\mathrm{id}}, S^{*} \sim\left(0, c^{S^{*}}(t, s), K^{S^{*}}(t, s, \cdot)\right)_{\mathrm{id}}$ be one-dimensional positive martingales, $S^{*}$ Markovian. Let $\beta=\beta(t) \geq 0$ be a barrier and $g$ be a payofffunction $g: \mathbb{R}_{+} \rightarrow \mathbb{R}_{+}$, such that $g$ is convex on (inf $\beta(t), \infty)$. Assume that $\mathcal{G}_{\text {out }}\left(t, S_{t}\right)$ is lower bounded and integrable, and that $\mathcal{G}_{\text {out }}(t, s) \in C^{1,2}\left([0, T] \times \mathbb{R}_{+}\right)$. Futher assume that that propagation of convexity holds i.e. $\mathcal{G}_{\mathrm{out}}(t, \cdot) \in \mathcal{F}_{\mathrm{cx}}$, and that the differential characteristics of $S, S^{*}$ are ordered

$$
\begin{aligned}
c_{t}^{S}(\omega) & \leq c^{S^{*}}\left(t, S_{t-}(\omega)\right), \\
\int_{(-1, \infty)^{d}} f\left(t, S_{t-}(\omega), x\right) K_{\omega, t}^{S}(d x) & \leq \int_{(-1, \infty)^{d}} f\left(t, S_{t-}(\omega), x\right) K_{t}^{S^{*}}\left(S_{t-}(\omega), d x\right),
\end{aligned}
$$

$\lambda \times Q$-a.e., for all $f:[0, T] \times \mathbb{R}_{+}^{d} \times(-1, \infty)^{d} \rightarrow \mathbb{R}$ with $f(t, s, \cdot) \in \mathcal{F}_{\mathrm{cx}}$ such that the integrals exist. Then the down-and-out barrier option prices are ordered

$$
E\left(g\left(S_{T}\right) \mathbb{1}_{\left\{S_{t}>\beta(t), \forall t \in[0, T]\right\}}\right) \leq E\left(g\left(S_{T}^{*}\right) \mathbb{1}_{\left\{S_{t}^{*}>\beta(t), \forall t \in[0, T]\right\}}\right) .
$$

Proof: We sketch the proof, which is based on the same approach as in the proofs of the comparison results of Section 3. Let $\tau_{\beta}(t):=\inf \left\{t>0: S_{t} \leq \beta\right\}$ denote the time at which $S$ first crosses the barrier $\beta$. Then it follows from Itô's formula and the Kolmogorov backward equation in (5.5) that

$$
\begin{aligned}
& \mathcal{G}_{\text {out }}\left(t \wedge \tau_{\beta}, S_{t \wedge \tau_{\beta}}\right) \\
& =\mathcal{G}_{\text {out }}\left(0, S_{0}\right)+M_{t \wedge \tau_{\beta}} \\
& +\int_{0}^{t \wedge \tau_{\beta}}\left\{\mathrm{D}_{s s}^{2} \mathcal{G}_{\text {out }}\left(u, S_{u-}\right)\left(c_{u}^{S}-c^{S^{*}}\left(u, S_{u-}\right)\right)\right. \\
& \left.\quad+\int_{0}^{t \wedge \tau_{\beta}}\left(\Lambda \mathcal{G}_{\text {out }}\right)\left(u, S_{u-} y\right)\left(K_{u}^{S}(d y)-K^{S^{*}}\left(u, S_{u-}, d y\right)\right)\right\} d u
\end{aligned}
$$

For $t=T$ holds $\mathcal{G}_{\text {out }}\left(T \wedge \tau_{\beta}, S_{T \wedge \tau_{\beta}}\right)=0$ on $\left\{T>\tau_{\beta}\right\}$. Therefore we obtain from (5.8) using the ordering assumptions in (5.6)

$$
\begin{aligned}
E \mathcal{G}_{\text {out }}\left(T \wedge \tau_{\beta}, S_{T \wedge \tau_{\beta}}\right) & =E \mathcal{G}_{\text {out }}\left(T \wedge \tau_{\beta}, S_{T \wedge \tau_{\beta}}\right) \mathbb{1}_{\left\{T \leq \tau_{\beta}\right\}} \\
& =E g\left(S_{T}\right) \mathbb{1}_{\left\{T \leq \tau_{\beta}\right\}} \\
& \leq \mathcal{G}_{\text {out }}\left(0, S_{0}\right)=E g\left(S_{T}^{*}\right) \mathbb{1}_{\left\{T \leq \tau_{\beta}^{*}\right\}}
\end{aligned}
$$

where $\tau_{\beta}^{*}=\inf \left\{t>0: S_{t}^{*} \leq \beta(t)\right\}$. This implies the inequality in (5.7).

Remark 5.2 Eriksson (2004, 2006) establishes the propagation of convexity condition $\mathcal{G}_{\text {out }}(t, \cdot) \in \mathcal{F}_{\text {cx }}$ for diffusion models for several types of single barrier options. He uses this property to derive upper and lower bounds for barrier options prices for stochastic volatility models $S \sim\left(0, c^{S}, 0\right)_{\text {id }}$ compared to diffusion models $S^{*} \sim\left(0, c^{S^{*}}(t, s), 0\right)_{\text {id }}$. For this case also the differentiability condition $\mathcal{G}_{\text {out }}(t, s) \in C^{1,2}$ is satisfied and thus Theorem 5.1 can be seen as an extension of Eriksson's results. Is remains however to investigate the propagation of convexity property for further types of barriers and in further models. 


\section{References}

[1] J. Barraquand and T. Pudet. Pricing of American path-dependent contingent claims. Math. Finance, 6(1):17-51, 1996.

[2] N. Bellamy and M. A. Jeanblanc. Incompleteness of markets driven by a mixed diffusion. Finance Stoch., 4:209-222, 2000.

[3] J. Bergenthum. Comparison of semimartingales and Lévy processes with applications to finanical mathematics. PhD thesis, University of Freiburg, 2005.

[4] J. Bergenthum and L. Rüschendorf. Comparison of option prices in semimartingale models. Finance Stoch., 10(2):222-249, 2006.

[5] J. Bergenthum and L. Rüschendorf. Comparison of semimartingales and Lévy processes. Annals of Probability, 35(1), 2007a.

[6] J. Bergenthum and L. Rüschendorf. Convex ordering criteria for Lévy processes. Advances Data Analysis Classification, 1:143-173, $2007 \mathrm{~b}$.

[7] R. Cont and E. Voltchkova. Integro-differential equations for option prices in exponential Lévy models. Finance Stoch., 9(3):299-325, 2005.

[8] R. Cont, P. Tankov, and E. Voltchkova. Option pricing models with jumps: Integrodifferential equations and inverse problems, 2004. http: / / www . mit.jyu.fi / eccomas 2004 / proceedings / pdf / 637.pdf.

[9] E. Ekström. Properties of American option prices. Stochastic Process. Appl., 114 (2):265-278, 2004.

[10] N. El Karoui, M. Jeanblanc-Picqué, and S. E. Shreve. Robustness of the Black and Scholes formula. Math. Finance, 8(2):93-126, 1998.

[11] J. Eriksson. Properties of European and American barrier options. Licentiate thesis, Uppsala universitet, 2004.

[12] J. Eriksson. Monotonicity in the volatility of single-barrier option prices. Int. J. Theor. Appl. Finance, 9(6):987-996, 2006.

[13] P. Franken and B. M. Kirstein. Zur Vergleichbarkeit zufälliger Prozesse. Math. Nachr., 78:197-205, 1977.

[14] A. A. Gushchin and E. Mordecki. Bounds for option prices in the semimartingale market models. Proceedings of the Steklov Mathematical Institute, 237:73-113, 2002.

[15] B. Hajek. Mean stochastic comparison of diffusions. Z. Wahrsch. Verw. Gebiete, 68 (3):315-329, 1985.

[16] V. Henderson. Price comparison results and super-replication: an application to passport options. Appl. Stoch. Models Bus. Ind., 16(4):297-310, 2000.

[17] V. Henderson and D. G. Hobson. Coupling and option price comparisons in a jump diffusion model. Stochastics and Stochastics Reports, 75(3):79-101, 2003. 
[18] D. G. Hobson. Robust hedging of the lookback option. Finance Stoch., 2(4):1329347, 1998.

[19] D. G. Hobson. Volatility misspecification, option pricing and superreplication via coupling. Ann. Appl. Probab., 8(1):193-205, 1998.

[20] J. Jacod and A. N. Shiryaev. Limit Theorems for Stochastic Processes. Springer, 2003.

[21] F. Jamshidian. The duality of optimal exercise and domineering claims. Preprint, 2006. http: / / wwwhome. math. utwente.nl/ jamshidianf.

[22] S. Janson and J. Tysk. Volatility time and properties of option prices. Ann. Appl. Probab., 13(3):890-913, 2003.

[23] A. Müller and D. Stoyan. Comparison Methods for Stochastic Models and Risks. John Wiley \& Sons, Ltd, 2002.

[24] H. Pham. Optimal stopping, free boundary, and American option in a jump-diffusion model. Appl. Math. Optim., 35(2):145-164, 1997.

[25] L. Rüschendorf. Stochastic ordering of risks, influence of dependence and a.s. constructions. In N. Balakrishnan, I. G. Bairamov, and O. L. Gebizlioglu, editors, Advances in Models, Characterizations and Applications, volume 180 of Statistics: Textbooks and Monographs. CRC Press, 2005.

[26] J. Večeř and M. Xu. The mean comparison theorem cannot be extended to the Poisson case. J. Appl. Probab., 41(4):1199-1202, 2004.

[27] X. Zhang. Analyse numérique des options américaines dans un modèle de diffusion avec des sauts. Doctoral dissertation, Ecole nationale des ponts et chaussées, Paris, 1994.

[28] X. Zhang. Some intersection theorems and minimax inequalities. J. Optim. Theory Appl., 94(1):195-207, 1997.

Jan Bergenthum

Mathematical Stochastics

Eckerstr. 1

79104 Freiburg

Germany
Ludger Rüschendorf

Mathematical Stochastics

Eckerstr. 1

79104 Freiburg

Germany

ruschen@stochastik.uni-freiburg.de 